Service social

\title{
La circularité du questionnement et des questions dans l'entrevue circulaire
}

\section{Thérèse Lane}

Volume 43, numéro 3, 1994

Intervention individualisée et empowerment

URI : https://id.erudit.org/iderudit/706668ar

DOI : https://doi.org/10.7202/706668ar

Aller au sommaire du numéro

\section{Éditeur(s)}

École de service social de l'Université Laval

ISSN

1708-1734 (numérique)

Découvrir la revue

Citer cet article

Lane, T. (1994). La circularité du questionnement et des questions dans l'entrevue circulaire. Service social, 43(3), 59-77.

https://doi.org/10.7202/706668ar
Résumé de l'article

Cet article présente une technique d'intervention très efficace en approche de court terme L'auteure situe d'abord le cadre théorique du modèle d'intervention élaboré par l'Équipe de milan dans lequel l'entrevue circulaire a été instaurée, puis elle rappelle les conditions sous-jacentes à la formulation des questions circulaires, soit la positions méta et la centration sur le processus. Vient ensuite l'explication d'un tableau synthèse reproduit à partir de deux diagrammes conçus par Karl Tomm. On y présente, de façon détaillée, tous les types de questions qui peuvent être formulées dans le cadre d'une entrevue circulaire, les différentes intentions que peut avoir le thérapeute en posant telles questions ainsi que les effets que chaque type de questions suscite chez le système-client et chez le système-intervenant. 


\section{La circularité du questionnement et des questions dans l'entrevue circulaire}

Thérèse LANE

Travailleuse sociale, consultante, formatrice et superviseure

La pratique professionnelle du travailleur social en intervention individuelle, conjugale et familiale reste encore profondément influencée par le modèle biomédical.

Si un bon nombre de travailleurs sociaux arrivent à sortir de I'ornière de la perspective individuelle et intrapsychique et à élargir la lecture du problème aux systèmes plus larges dans lesquels il se manifeste, on se bloque, dans certaines situations, à une analyse tout aussi linéaire. Au lieu de mettre en cause l'individu, on met alors en cause la dynamique familiale, ignorant totalement l'implication des autres systèmes. On se donne le mandat ou le rôle de travailler sur la famille. On va même jusqu'à se définir comme l'avocat du jeune, la partie adverse étant les parents. En agissant ainsi, on ne fait qu'amplifier l'escalade de la disqualification des parents.

Petit à petit, l'image d'un système complètement démuni prend toute la place. Il devient justifié qu'on leur montre comment faire et qu'on leur propose des solutions. On parle de non-collaboration 
et de résistance de la famille. On finit par attribuer à la famille la responsabilité du non-changement: "Ça ne peut pas faire autrement, cette famille a un système tellement rigide " ou encore "Avec des parents non volontaires, comment pourrais-je amorcer un changement?»

L'individu, le couple, la famille ou le référent se présente avec une perception très linéaire du problème voulant que tout effet soit le produit $d^{\prime}$ une cause. Par exemple, les problèmes de comportement de I'adolescent pourraient être causés par la grande rigidité des parents ou encore par le fait qu'ils ne lui donnent pas suffisamment $\mathrm{d}^{\prime}$ 'affection. Cette compréhension du problème est le terrain sur lequel le système-client cherche à amener l'intervenant. La façon dont est posé le problème définit les limites dans lesquelles on pourra le résoudre.

Comme l'exprime J.A. Malarewicz, «Le processus thérapeutique peut être décrit comme étant à la fois un processus de déconstruction et de construction. Ces deux mouvements étant simultanés» (1992:61).

La déconstruction et la construction visent la remise en question des points de repère habituels de la famille, tout particulièrement ceux qui concernent la compréhension du problème présenté. II s'agit de reconstruire la réalité de la famille de telle manière que sa relation au problème présenté ne peut plus être la même. On démonte une perception de la réalité tout en proposant une autre perception de cette même réalité. II s'agit des mêmes faits, mais reliés de telle façon qu'ils «prennent un sens» différent.

Le symptôme ou le problème, d'abord présenté en termes de caractéristiques individuelles, est transformé en description relationnelle. C'est ainsi que des influences réciproques jusque-là méconnues sont mises en évidence. Chacun perçoit l'implication de l'autre en même temps que la sienne dans la séquence interactionnelle.

Ce processus de déconstruction et de construction vient "dépathologiser», voire "dépsychologiser » la perception qu'a la famille de la situation-problème. Le déséquilibre ainsi introduit vient bloquer leurs modèles habituels de solution de problème et les ouvre, du même coup, à des avenues nouvelles. Pour enclencher ce processus de déconstruction et de construction, l'intervenant doit adopter un point de vue systémique, il doit s'intéresser aux mouvements circulaires qui existent entre les différents membres du système familial et avec les autres systèmes en cause dans la situationproblème (école, centre d'accueil, CLSC, etc.). C'est cette optique qui sous-tend I'entrevue circulaire dont l'équipe de Milan (Boscolo, Cecchin, Prata) a été I'instigatrice, avec Mara S. Palazzoli en tête. 
L'entrevue circulaire comporte deux aspects principaux, soit I'interrogation circulaire et les questions circulaires. L'interrogation circulaire désigne le processus interactif d'entrevue pris dans son ensemble. Durant cette entrevue I'intervenant pose des questions inspirées par les réponses de la famille, celle-ci répondant suivant les questions posées par I'intervenant. Quant aux questions circulaires, elles sont désignées ainsi du fait qu'elles explorent la circularité existant au sein du système familial et avec les autres systèmes en cause dans la situation-problème.

Karl Tomm, de l'université de Calgary, a repris le questionnement circulaire de Milan et en a fait la base d'une approche qu'il appelle interventive questioning. Dans une série d'articles dont les références sont présentées dans la bibliographie il décrit son approche de façon remarquable. Citons, tout particulièrement, le texte paru dans les Cahiers critiques de thérapie familiale et de pratiques de réseaux, dans lequel il présente de façon détaillée et vivante toute une série de questions réflexives susceptibles de mobiliser les capacités d'autoguérison de la famille.

Plusieurs auteurs ont contribué à perfectionner la théorie et la technique du questionnement circulaire, dont Peggy Penn, de I'Institut Ackermann à New York. S'inspirant des mots clés (métaphores) de la famille, celle-ci compare ce qui est dit avec les comportements analogiques des différents membres du système.

\section{CADRE THÉORIQUE DES QUESTIONS CIRCULAIRES}

Le concept de circularité caractérise les modèles d'interaction qui s'établissent entre les différents systèmes, tout comme il caractérise également les modèles d'interaction entre les composantes $d^{\prime} u n$ même système.

Par exemple, tous les membres d'une même famille sont engagés dans des échanges constants dans lesquels chacun influence les autres et ce, de façon réciproque. Après un certain temps, on ne peut reconnaître le point de départ ou la cause première au comportement d'un de ses membres.

Le même phénomène se produit dans la rencontre de différents systèmes. Dans une relation d'aide, le système-client et le systèmeintervenant s'engagent dans des échanges continus dans lesquels chacun influence l'autre de façon réciproque. Et chaque comportement est à la fois cause et effet. 
Cette notion de circularité a été élaborée par Bateson qui utilisait ce terme pour décrire l'organisation récursive qui caractérise, disait-il, les formes vivantes.

S'inspirant de cette notion présentée par Bateson, l'équipe de Milan a bâti le modèle d'intervention circulaire qui utilise, comme technique principale $d^{\prime}$ intervention, les questions circulaires.

Alors que le modèle «structural » de Minuchin se fonde sur la structure du système et que le modèle stratégique de Haley se centre davantage sur le fonctionnement du système, le modèle de l'école de Milan met surtout l'accent sur les croyances, sur les schémas de fonctionnement et de changement, tout en tenant compte de la structure et du fonctionnement du système.

Ce tournant fut en partie influencé par la propre croyance constructiviste de Bateson. Celui-ci affirme que dans les organismes vivants, le genre de prémisses abstraites qui concernent la survie sont établies à un niveau profond de structure. Ainsi, plutôt que de viser à changer de façon directe les structures familiales, les patterns interactionnels et autres, l'équipe milanaise se concentre d'abord sur les idées, les valeurs, les croyances qui maintiennent en place des modèles de comportements inadéquats et suscitent le malaise présenté.

Dans I'élaboration de ce modèle, on commence à voir un effet de la cybernétique du second ordre sur la pratique de la thérapie familiale.

On y considère les systèmes principalement "évolutifs », plutôt que «homéostatiques». La famille y est définie comme un système en perpétuel changement, fonctionnant de façon circulaire, selon les schémas habituels issus de la compréhension du monde. Quant au problème, il se présente quand le schéma habituel ne correspond plus aux possibilités des relations familiales dans un contexte changé.

L'équipe de Milan a défini trois principes de base qui guident la conduite de l'entrevue par le thérapeute: la neutralité, la formulation d'hypothèses et la circularité.

La neutralité est la position du thérapeute qui, dans la phase d'exploration, accepte les solutions jusqu'ici mises en œuvre par la famille comme les seules possibles, en raison des croyances, des patterns et de l'accès aux ressources. Son rôle est celui d'un stimulant pour aider la famille à sortir d'une impasse dans laquelle sa capacité auto-organisatrice s'est perdue.

Quant à I'hypothèse, elle "est une supposition non prouvée, acceptée à l'essai pour servir de base à une investigation ultérieure, dont on peut obtenir une vérification ou une réfutation» (1988). 
Elle sert de point de départ et occupe une position centrale dans le travail d'investigation. L'hypothèse guide la nouvelle prise d'information, au moyen de laquelle elle sera confirmée, réfutée ou modifiée. II serait juste de dire que les hypothèses introduisent de I'organisation dans l'entrevue. Une hypothèse circulaire introduira un ordre systémique, alors qu'une hypothèse linéaire amènera un ordre analytique. Il va sans dire que le thérapeute doit privilégier des hypothèses se rapportant à la fonction relationnelle du système.

Par ailleurs, il existe toujours le risque qu'un intervenant puisse tenir à une hypothèse à un point tel que celle-ci en devienne un croyance. Lorsque cela se produit, I'intervenant ne se situe plus dans une perspective systémique. En effet, s'il persiste à chercher la confirmation de sa supposition, il ne répond pas à la rétroaction circulaire du système thérapeutique. Même si le contenu de son hypothèse est circulaire en ce qui concerne le système-client, I'intervenant demeure linéaire en ce qui a trait à l'interaction entre le système-client et lui-même.

L'équipe de Milan définit ainsi le principe de la circularité:

Nous entendons par circularité la capacité du thérapeute à mener son enquête en se basant sur la rétroaction obtenue de la famille en réponse à l'information qu'il sollicite des relations familiales et, par conséquent, à propos de la différence et du changement (1988).

Cette circularité se concrétise dans l'interrogation circulaire et dans les questions circulaires. L'interrogation circulaire englobe non seulement les questions posées, mais surtout les effets qu'elles suscitent et chez la famille et chez l'intervenant qui, à partir des réponses, est amené à poser de nouvelles questions. La méthode alors utilisée, ce sont les questions circulaires. Une question dite circulaire est formulée de telle façon qu'elle met en relation deux ou trois données qui ont été présentées par la famille. En les mettant en relation dans une même question, ces différentes données apportées ici et là par la famille se trouvent transformées en information nouvelle. Par exemple: "Les querelles avec votre conjoint étaient-elles plus fréquentes avant ou après qu'il présente des problèmes cardiaques?».

En changeant ainsi les données en information, de nouvelles significations sont introduites dans le système. Pour ce faire, I'intervenant travaille avec deux points propres à ce modèle:

1. L'information réside dans les différences.

2. C'est dans son contexte qu'un comportement trouve sa signification. 
L'objectif visé par les questions circulaires est d'amener les membres du système familial à se communiquer de nouvelles informations et d'acquérir une perception différente de leur système d'interaction.

Aux trois grands principes définis par l'équipe de Milan, Karl Tomm en ajoute un quatrième: "l'élaboration de stratégies». C'est ici qu'il faut de nouveau souligner l'apport précieux de Karl Tomm. Cet expert en approche systémique a poussé plus loin la notion des questions circulaires élaborée par l'équipe de Milan. II a d'abord identifié les principaux types de questions formulées tout au long d'une entrevue à l'aide d'observations et d'expérimentations, puis il les a présentées sous forme de deux diagrammes en distinguant les questions linéaires, circulaires, réflexives et stratégiques (voir annexes A et B). Vous en trouverez la présentation et l'explication après avoir pris connaissance des conditions sous-jacentes à l'entrevue circulaire.

\section{CONDITIONS SOUS-JACENTES À L'ENTREVUE CIRCULAIRE}

L'entrevue circulaire suppose l'application des trois principes de base du modèle d'intervention élaboré par l'équipe de Milan. Elle implique également l'intégration des conditions sous-jacentes, soit la position méta et la centration sur le processus.

\section{Position méta}

Méta signifie: «au-dessus », "à un autre niveau ». Se placer en position méta signifie se placer au-dessus, à un autre niveau, c'est-à-dire se voir comme «distinct» de la situation dans laquelle on se trouve tout en se voyant «en relation avec ». D'après Jacqueline Prud'homme, la position méta permet de libérer un espace intérieur afin de nous positionner, puis de nous dégager s'il y a lieu d'un mandat inapproprié et d'utiliser les moyens d'intervention qui conviennent.

Le travailleur social est comme un navigateur naviguant entre les systèmes impliqués. S'il ne tient pas sa boussole qui est pour moi la position méta, il est ballotté entre les différentes composantes d'un système ou entre les différents systèmes. II perd sa visibilité et n'arrive plus à se positionner... Quel espace intérieur arrivons-nous à avoir pour pouvoir être en résonance avec les autres, mais aussi pour pouvoir penser? Sans cet espace, pas moyen de penser, pas moyen de créer, pas moyen de jouer avec ses pensées ni avec celles des autres (1990). 
Elle nous présente la "position méta » comme faisant partie de son quotidien, dans le cadre de sa pratique professionnelle. Signalant le danger qui guette tout travailleur social de se définir uniquement par rapport aux autres, elle propose la position méta en tant que moyen de se définir et de devenir créateur.

Quant à Mara Selvini Palazzoli, elle affirme:

Nous croyons que le thérapeute ne peut être efficace que dans la mesure où il est capable d'obtenir et de maintenir un niveau différent (méta niveau) de celui de la famille (1988).

À son avis, ce méta niveau procure à l'intervenant une marge de manœuvre pour amorcer une stratégie visant à recueillir l'information qui lui sera nécessaire afin d'intervenir sur les enjeux de la demande. Autrement, il se lie les mains et se voit imposer une façon de fonctionner, issue des stratégies déjà en cours.

Tant M. Palazzoli que J. Prud'homme présentent la position méta comme un outil qui amène l'intervenant à enclencher un processus de changement au lieu de demeurer un élément qui sert au maintien du problème présenté.

En effet, cette espèce de position "parapluie » nous permet de prendre une distance tout en restant à proximité. Je suis là sans être là, uniquement. Cette mise à distance nous permet une plus grande présence et une plus grande écoute de ce qui se vit tout de suite au sein du système de changement.

$C^{\prime}$ est à partir de la position méta que l'intervenant devient en mesure de formuler des questions circulaires et d'enclencher un processus de changement de perception, puis d'action.

\section{Centration sur le processus}

En position méta, l'intervenant se centre sur le processus plutôt que sur le contenu.

La famille se présente en définissant fortement son problème comme étant celui d'un individu. Les dimensions du problème sont réduites pour les centrer sur les difficultés d'un seul de ses membres. Par conséquent, le danger qui guette le travailleur social est le souci du contenu, alors qu'il n'est que l'instrument qui véhicule le processus.

Pour être efficace, le travailleur social doit plus porter attention à la façon dont le message est transmis, à qui il est transmis et à l'effet qu'il produit qu'au message lui-même. S'il demeure centré sur le contenu il risque de glisser facilement dans le piège de la recherche de solutions. 
Virginia Satir, pionnière de l'approche systémique, a été une des premières à affirmer que les intervenants ne devraient pas se soucier de ce que devrait faire la famille, mais qu'ils devraient plutôt se consacrer à créer de nouvelles possibilités pour les membres de cette famille. Elle insistait sur le fait que ce qui compte $n^{\prime}$ est pas tant le problème en lui-même que la manière dont les membres d'une famille le construisent.

L'entrevue circulaire devient à cet égard un outil qui nous permet de nous interroger sur la manière dont les membres d'une famille perpétuent le problème présenté. Pour ce faire, la position méta et la "centration sur le processus » représentent des conditions préalables. Ces deux conditions sont d'ailleurs dans une interdépendance circulaire en ce sens qu'en se plaçant en position méta on se centre automatiquement sur le processus ou, vice versa, qu'en se centrant sur le processus on se place automatiquement en position méta.

\section{EXPLICATION DU TABLEAU SYNTHÈSE}

Afin de faciliter l'identification des divers types de questions possibles, je présente un tableau synthèse dans lequel on trouve tous les types de questions, les différentes intentions que peut avoir le thérapeute en posant telles questions, de même que les effets que chaque type de questions suscite chez le système-client et chez le système-intervenant. Ce tableau synthèse a été reproduit à partir des deux diagrammes conçus par Karl Tomm (voir annexes A et B).

Ainsi qu'il a été mentionné précédemment, Karl Tomm a poussé plus loin la notion des questions. II a d'abord catégorisé les différentes questions qu'un intervenant pose, en entrevue, à partir de deux dimensions de base: la cible visée (visent la description ou visent l'intervention) et le type d'hypothèses formulées (hypothèses linéaires ou hypothèses circulaires).

Comme le processus a avantage à se dérouler de la gauche vers la droite, je présente d'abord les questions linéaires, pour aller vers les questions circulaires, puis réflexives et finalement stratégiques.

Les questions classées dans la partie gauche inférieure et supérieure du diagramme (linéaires et circulaires) sont censées viser en priorité un changement à opérer chez l'intervenant, c'est-à-dire dans sa propre compréhension du système-client, alors que les questions de la partie droite supérieure et inférieure (réflexives et stratégiques) visent plutôt un changement à effectuer chez le système-client. 


\section{Tableau synthèse}

Expérimentation de la démarche du questionnement circulaire

\section{Hypothèses circulaires}

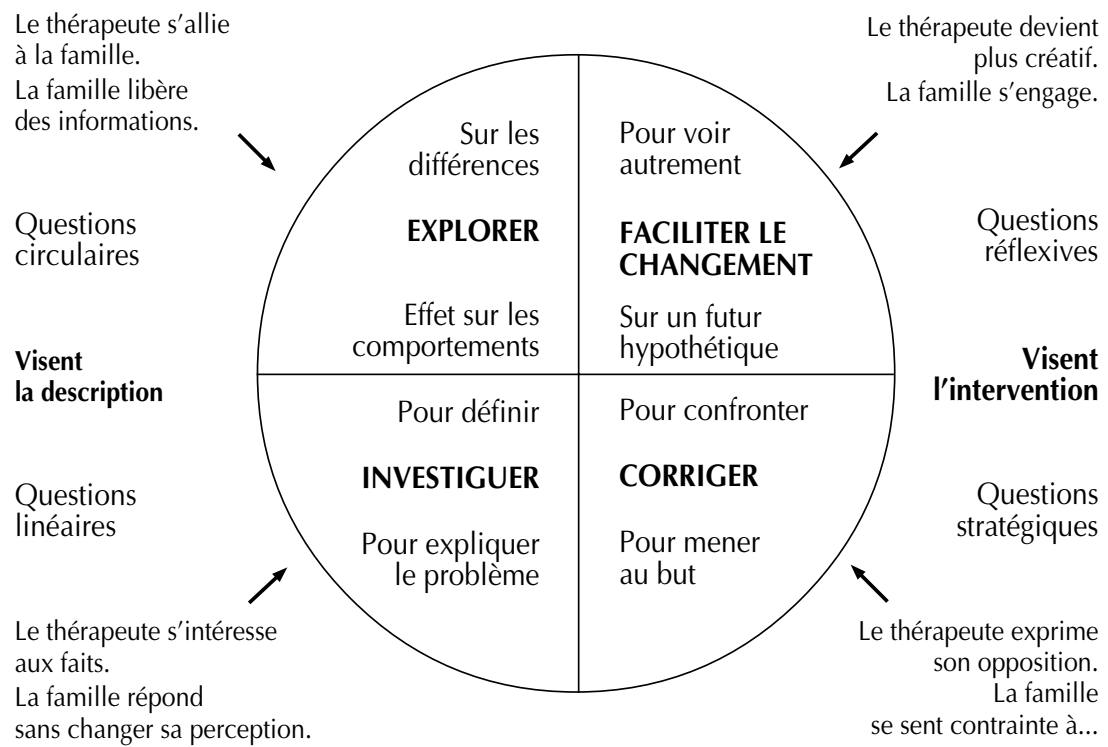

Hypothèses linéaires

Tiré de : Figure 1 : Karl Tomm, "Circumplex relationships among alternative modes of enquiry », Family Process, vol. 27, mars 1988, p. 8.

Figure 2 : Karl Tomm, "Predominant intent and idiosyncratic effects of different questions », Family Process, vol. 27, mars 1988, p. 13.

Les questions classées dans la partie inférieure gauche et droite du diagramme (linéaires et stratégiques) sont censées être formulées à partir d'hypothèses linéaires. Dans une perspective linéaire, l'intervenant cherche la cause, il interroge selon une perception de cause à effet, soit :

$$
A+B=C
$$

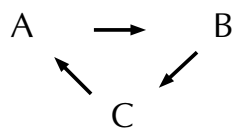


Le comportement de A devient la cause du comportement de B, tout comme le comportement de B devient la cause du comportement de $C$ et ainsi de suite.

À partir des hypothèses linéaires, on trouve d'abord, dans le quadrant inférieur gauche, les questions linéaires (pour définir le problème et pour expliquer le problème) qui amènent l'intervenant à s'intéresser aux faits, et la famille à répondre sans changer sa perception. Par ses questions, l'intervenant se montre intéressé à connaître les points de vue des membres de la famille et leurs sentiments en ce qui a trait à la définition du problème, son origine, ses causes et ses effets. Ce type de questions amène la famille à rapporter les faits tels qu'elle les perçoit. Les questions étant formulées de façon linéaire, les membres de la famille ne sont pas habituellement amenés à remettre en question ou à changer leur façon de voir habituelle. C'est pourquoi ce type de questions rend plus facile la prise de contact. En formulant ces deux types de questions, l'intention du thérapeute est d'investiguer.

Par ailleurs, les questions dans la partie supérieure gauche et droite (circulaires et réflexives) risquent plus d'être formulées à partir d'hypothèses circulaires.

Dans une perspective circulaire, l'intervenant vise à faire ressortir la réciprocité des composantes du système en question, soit:

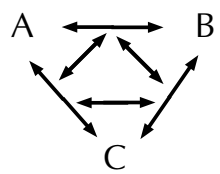

$A$, B et $C$ sont engagés dans des échanges constants dans lesquels chacun influence l'autre de façon réciproque. On ne peut identifier de point de départ ou de cause première au comportement de l'un ou de l'autre, car chaque comportement est à la fois cause et effet. De même qu'on ne peut reconnaître de cause première à la relation entre I'un et I'autre, car toute modification de la relation entre deux membres vient influer sur la relation entre les autres.

Par conséquent, s'il vise à modifier sa propre compréhension du système-client, l'intervenant posera des questions linéaires ou circulaires selon les hypothèses auxquelles il travaille. S'il vise plutôt à inciter la famille à voir sa situation d'une manière différente, pour agir ensuite de façon différente, il posera des questions réflexives ou stratégiques selon ses hypothèses ou la nature du changement qu'il espère déclencher dans la famille. 
À partir d'hypothèses circulaires, on trouve, d'une part, dans le quadrant supérieur gauche, les questions circulaires (sur les différences et effets sur les comportements). En formulant ces deux types de questions, l'intervenant a l'intention d'explorer.

Pour arriver à formuler des questions circulaires, l'intervenant est amené à établir un lien entre les différents éléments présentés, de façon détachée, par les différents membres de la famille. Cet exercice l'amène à s'allier au système-famille à un autre niveau dans le but d'en acquérir une connaissance de plus en plus globale et de plus en plus systémique. Par leur formulation, ces questions amènent le système-famille à libérer un type d'informations qu'il ne s'était fort probablement jamais partagées auparavant et à commencer à remettre en question sa façon de voir habituelle.

D'autre part, on trouve, dans le quadrant supérieur droit, les questions réflexives (pour voir autrement et celles sur un futur hypothétique) qui amènent l'intervenant à faire appel à sa créativité de façon encore plus particulière et qui conduisent la famille à s'engager. En formulant ces deux types de questions, l'intervenant a I'intention de faciliter le changement.

Finalement, à partir d'hypothèses linéaires, on trouve, dans le quadrant inférieur droit, les questions stratégiques (pour confronter et pour mener au but), qui amènent l'intervenant à exprimer son opposition et qui suscitent une pression et un effet de contrainte chez la famille. En formulant ces deux types de questions, I'intention du thérapeute est de corriger.

Comme je l'exprimais précédemment, ce type de questions étant censées être formulées en fin de processus, c'est-à-dire après qu'on a posé des questions linéaires, circulaires puis réflexives, il peut être très opportun que l'intervenant décide d'exprimer ouvertement son opposition sous forme d'une question stratégique. II vise, par une telle question, à ce que les membres de cette famille se sentent plus fortement contraints à reproduire les mêmes comportements aux conséquences très négatives et très néfastes pour chacun. L'avantage d'une intervention prenant la forme d'une question stratégique est que l'intervenant n'a pas besoin de défendre la position sousentendue par la question, comme il le ferait s'il prononçait une affirmation. Après avoir posé une question stratégique, il décide, selon la réaction de la famille, s'il y a lieu ou non de poursuivre avec le même type de questions. Il risque moins de cette façon d'aboutir à une impasse.

Chacun des types de questions qui se retrouvent dans toute la partie gauche du tableau (inférieure et supérieure) vise la description 
du problème ou, plus précisément, vise la modification chez l'intervenant de sa propre perception du système-client ou de sa propre définition du problème. Le terme «modifier » est juste en ce sens que I'intervenant se fabrique, de façon consciente ou pas, une image du système-client, dès la première conversation téléphonique. C'est à partir de cette image qu'il procède à la première entrevue.

Les questions linéaires l'amènent à connaître les perceptions du système-client en ce qui concerne la définition et l'explication du problème; les questions circulaires lui permettent ensuite $d^{\prime}$ en acquérir une perception plus globale et plus systémique. Chaque type de questions se retrouvant dans la partie droite du tableau (supérieure et inférieure) vise I'intervention ou plus précisément vise à amener le système-client à acquérir de nouvelles possibilités de significations et d'actions.

Il faut préciser qu'il ne s'agit pas ici d'un processus linéaire dans lequel I'intervenant viserait d'abord à saisir tous les éléments du problème et à le comprendre une fois pour toutes pour être plus en mesure de vendre ensuite au système-client la perception qu'il a acquise de sa situation-problème et de lui proposer des solutions.

Au contraire, il s'agit d'un processus circulaire et dynamique au cours duquel le système (intervenant et client) s'engage dans une démarche qui vise autant à modifier la perception de l'intervenant que celle du système-client. Ces deux objectifs sont poursuivis et atteints dans un processus continu d'échanges. Dans ce processus I'un vient susciter un effet chez l'autre qui, à la suite de cette nouvelle information, est amené à réagir d'une telle façon qui amène l'un à... et ainsi de suite, la boucle continue.

Dans le contexte d'une entrevue, rares sont les questions qui peuvent typiquement n'appartenir qu'à une seule catégorie. La plupart peuvent s'identifier à deux catégories à la fois.

Comme I'exprime Karl Tomm, certaines questions pourraient être classées dans n'importe quel quadrant du diagramme, parce qu'elles contiennent des éléments de linéarité et de circularité et qu'elles pourraient viser le changement de perception autant chez l'intervenant que chez le système-client.

Cependant, certains types de questions peuvent appartenir de façon plus spécifique à une catégorie. Par exemple, appartiennent à la catégorie des questions linéaires:

1. les questions qui visent à définir le problème ; (Quel problème vous amène ici? Avez-vous d'autres symptômes?)

2. celles qui visent à expliquer le problème.

(Depuis quand êtes-vous déprimé?) 
Quant aux questions circulaires, elles comprennent, entre autres :

1. les questions qui portent sur les différences de catégories;

2. les questions qui portent sur les différences temporelles concernant le passé et le présent ;

3. les questions qui portent sur l'effet produit sur le comportement.

Les questions portant sur les différences de catégories explorent:

- les différences descriptives entre les personnes;

(Qui est le plus inquiet?)

- les différences dans les relations;

(De qui le père est-il le plus proche?)

- les différences d'idées, de perceptions, de valeurs et de croyances.

(Qu'est-ce qui, à ton avis, effraie le plus tes parents quand tu rentres si tard?)

Les questions portant sur les différences temporelles explorent les différences entre:

- deux événements du passé ;

(Les querelles étaient-elles plus fréquentes avant ou après la crise cardiaque?)

- le passé et le présent.

(La mère était-elle plus proche de Nicole lorsqu'elle était plus jeune ou est-ce maintenant?)

Les questions qui portent sur l'effet produit sur le comportement explorent les liens entre des actions précises qui se produisent au cours d'un processus d'intervention entre les membres d'un système. Afin de faire ressortir la répétition circulaire du pattern de fonctionnement, l'intervenant doit continuer à explorer les liens de comportement jusqu'à ce que les séquences reviennent à leur point de départ et se répètent.

Par exemple:

Que faites-vous quand votre conjoint déprime?

Quand votre conjointe se fâche, comment réagissez-vous?

Lorsque vous vous fâchez et que votre conjoint fuit, que font les enfants?

Que fait votre conjoint quand vos enfants viennent s'occuper de vous? 
Après ces quatre questions, le pattern peut être établi : Monsieur déprime, Madame se fâche. Monsieur fuit. Les enfants viennent s'occuper de Madame. Monsieur déprime et ça recommence.

Comme questions réflexives, on retrouve plus particulièrement:

1. les questions qui portent sur les différences temporelles, plus spécifiquement celles orientées vers le futur, appelées hypothétiques;

2. les questions qui amènent le système-client à se placer dans une position d'observateur, comme les questions triadiques.

Les questions qui portent sur les différences temporelles explorent les différences entre :

- le passé et le futur;

(Si vous n'étiez pas retourné aux études, croyez-vous $q u$ 'il serait plus probable que vous viviez encore en couple, dans deux ans d'ici?)

- le présent et le futur;

(Qu'arriverait-il de la relation entre votre père et les autres enfants si votre mère devait être retenue à son travail tous les soirs?)

- deux événements futurs.

(Si votre conjointe décidait d'aider vos enfants à mieux percevoir vos interactions, comment pourrait-elle le faire?)

Ces questions orientées vers le futur sont particulièrement utiles pour stimuler le système-client à considérer de nouvelles possibilités de signification et d'action.

Quant aux questions triadiques, elles sont plus manifestement circulaires du fait qu'elles sont adressées à une troisième personne et qu'elles portent sur la relation entre deux autres personnes.

- Quand votre sœur rentre plus tard que l'heure prévue, qu'est-ce que cela vient changer dans la relation entre votre père et votre mère?)

En ce qui concerne les questions stratégiques, elles renvoient:

1. aux types de questions pour "confronter»; (Ne voyez-vous pas jusqu'à quel point votre comportement préoccupe votre conjointe?)

2. aux types de questions pour mener au but. (Quand allez-vous prendre votre vie en main et commencer à chercher du travail ?) 
Chacune de ces catégories a son importance selon l'étape où I'on est rendu dans le processus et selon le but visé.

Le processus aurait avantage à se dérouler de la gauche vers la droite, c'est-à-dire en commençant par des questions linéaires, puis en allant vers des questions circulaires, puis réflexives et finalement stratégiques. Mais il ne s'agit pas de le faire de façon exclusive et stéréotypée. Il est parfois opportun d'intercaler quelques questions linéaires entre des questions réflexives et circulaires, par exemple.

Premièrement, comme la plupart des clients arrivent avec une perception plutôt linéaire de leur situation-problème, le fait de commencer l'entrevue par des questions linéaires permet à l'intervenant de créer le contact et de rejoindre les personnes sur le plan de leur façon de voir et de fonctionner. Les demandes directes au sujet des pensées et des sentiments personnels ainsi que de leurs causes apportent des informations qui constituent la base nécessaire à I'acquisition d'une compréhension systémique.

Deuxièmement, il est pertinent de poursuivre avec des questions circulaires qui, en faisant appel à la circularité qui existe au sein du système-client, amènent l'intervenant à modifier la compréhension qu'il en a pour la rendre plus globale et systémique.

Troisièmement, les questions réflexives basées sur la perspective de l'observateur, invitant le système-client à se placer en position méta et à remarquer des patterns qu'il n'a peut-être pas distingués jusqu'ici l'amènent à explorer de lui-même de nouvelles possibilités de significations et d'actions et à amorcer le changement.

Quatrièmement, les questions stratégiques amènent le systèmeclient à entrevoir des pistes de changement qu'il n'aurait peut être pas reconnues de lui-même. Posées dans un contexte approprié, elles peuvent être très efficaces. Dans le cadre de la démarche décrite par Karl Tomm, il convient de poser les questions stratégiques uniquement dans les situations où les trois autres types de questions $n^{\prime}$ ont pas suffi à susciter le changement souhaité.

Il importe par ailleurs d'utiliser les différentes catégories de questions avec une certaine prudence.

Tout comme il est aussi important de recourir à des questions formulées à partir d'hypothèses linéaires (linéaires et stratégiques) qu'à des questions formulées à partir d'hypothèses circulaires (circulaires et réflexives) selon l'étape où l'on est rendu, le but visé et la réaction du système-client, il serait tout aussi inapproprié de se limiter à des questions formulées à partir d'hypothèses linéaires ou à des questions formulées à partir d'hypothèses circulaires. 
Par exemple, dans certaines situations, il arrive qu'on se limite aux questions linéaires (de définition et d'explication du problème). On ne prend pas le recul nécessaire qui permettrait de mettre en lien les éléments présentés par le système-client et de les traduire sous forme de questions circulaires puis réflexives. Enfermé dans une perception linéaire du problème, on passe directement aux questions stratégiques (pour confronter et pour mener au but). Posées dans tel contexte, elles risquent d'attaquer et même de disqualifier le systèmeclient au lieu d'introduire une simple confrontation. À la résistance manifestée par l'intervenant, le système-client répondra par la résistance, puis ce sera l'impasse.

Dans d'autres situations, il arrive qu'on se précipite dans des questions circulaires sans avoir d'abord pris le temps de rejoindre le système-client dans sa façon de voir et de fonctionner au moyen des questions linéaires qui font appel à des éléments fondamentaux en ce qui a trait à la définition du problème. Cette catégorie de questions qui, posées en temps opportun, amènent le système-client à libérer des informations risquent au contraire de le heurter et de ne susciter que de la résistance.

Inutile de dire qu'il serait tout aussi inapproprié de n'utiliser que cette technique d'intervention tout au long d'une entrevue. L'utilisation des questions circulaires n'empêche pas pour autant le recours aux autres techniques circulaires d'intervention, telles que génogramme, sculpture, métaphore... 


\section{Références bibliographiques}

BAteson, G.I. (1984). La nature et la pensée. Paris: Le Seuil, p. 235.

BAtesOn, G. et M.C. BAteson (1989). La peur des anges. Le Seuil, p. 294.

BAteson, G. (1977, 1980). Vers une écologie de l'esprit, tomes 1 et 2. Paris : Le Seuil.

EVEQUOZ, Grégoire (1984). Le contexte scolaire et ses otages. Paris: ESF, p. 140.

MALAREWICZ, J.A. (1992). Quatorze leçons de thérapie stratégique. Paris: ESF.

PRUD'HOMME, Jacqueline (1990). "Le travailleur social et ses paradoxes", Bulletin de nouvelles CPTSQ, juin, $\mathrm{n}^{\circ}$ 38:21-26.

SElVINI, Matteo et SELVINI PALAZZOLI, M. (1988). Histoire d'une recherche. Paris: ESF, p. 225.

Selvini PAlazzolı, M. et al. (1984). Dans les coulisses de l'organisation, stratégie et tactique. Paris: ESF.

SElVINI PALAZZOLI, M. (1980). La magie sans magie. Paris: ESF, p. 145.

SElviNi PALAZZOLI, M. et al. (1982). "Hypothétisation - Circularité Neutralité », Thérapie familiale, Genève, vol. 3, n 3: 117-132.

SEYWERT, Ferdinand (1993). "Le questionnement circulaire», Thérapie familiale, Genève, vol. 14, no $1:$ 73-88.

SPECTOR-DUNSKY, L. (1985). "Du linéaire à la circularité », Systèmes humains, vol. 1, $\mathrm{n}^{\circ} 2: 51-59$.

TOMM, K. (1985). "Interventive Interviewing : Part I. Strategizing as a Fourth Guideline for the Therapist», Family Process, 26:3-13.

TOMM, K. (1985). "Interventive Interviewing: Part II. Reflexive Questioning as a Means to Enable Self Healing », Family Process, 26 : 153-183.

TOMM, K. (1988). "Interventive Interviewing: Part III. Intending to Ask Lineal circular, Strategic, or Reflexive Questions », Family Process, 27 : 1-16.

TOMM, K. (1986). "La thérapie systémique: vue d'ensemble de l'approche de l'École de Milan», Systèmes humains, vol. 2, nº $1:$ 9-61.

TOMM, K. (1991). "Les questions réflexives, instruments d'auto-guérison», Cahiers critiques de thérapie familiale et de pratiques de réseaux, Privat, vol. $13: 199-227$.

VARELA, F. (1991). "Les multiples figures de la circularité », Cahiers critiques de thérapie familiale et de pratiques de réseaux, Privat, vol. 9: 45-49. 


\section{Annexe A}

\section{Circumplex relationships among alternative modes of enquiry}

\section{Circular Assumptions}

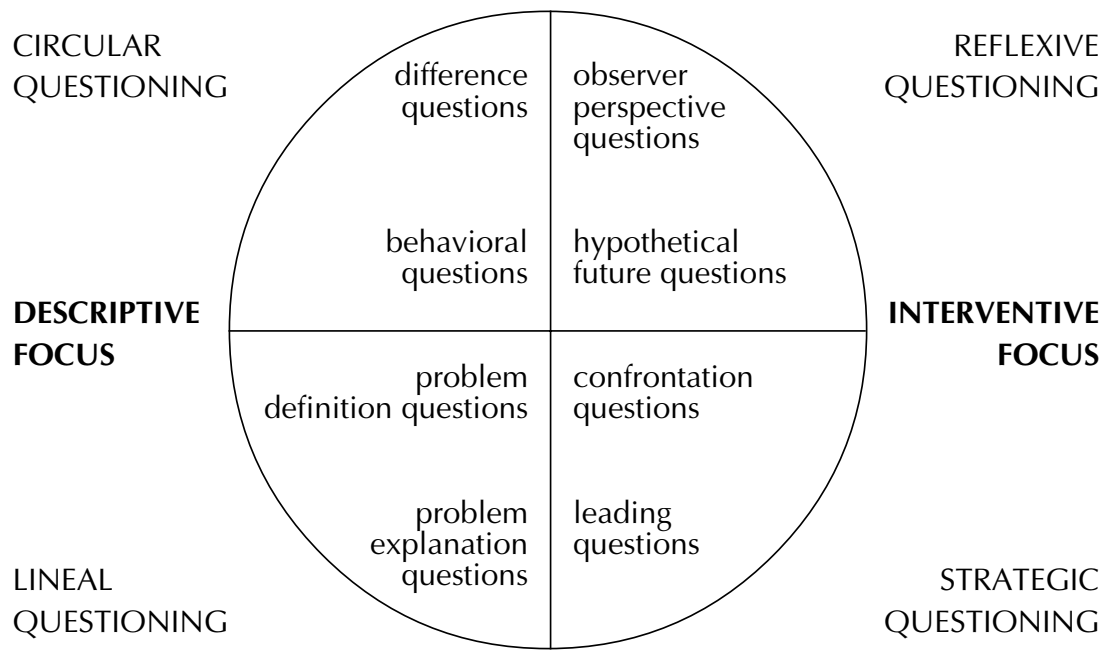

Linear Assumptions

Tiré de Karl Tomm, Family Process, vol. 27, mars 1988, p. 6. 


\section{Annexe B}

\section{Predominant intent and idiosyncratic effects of different questions}

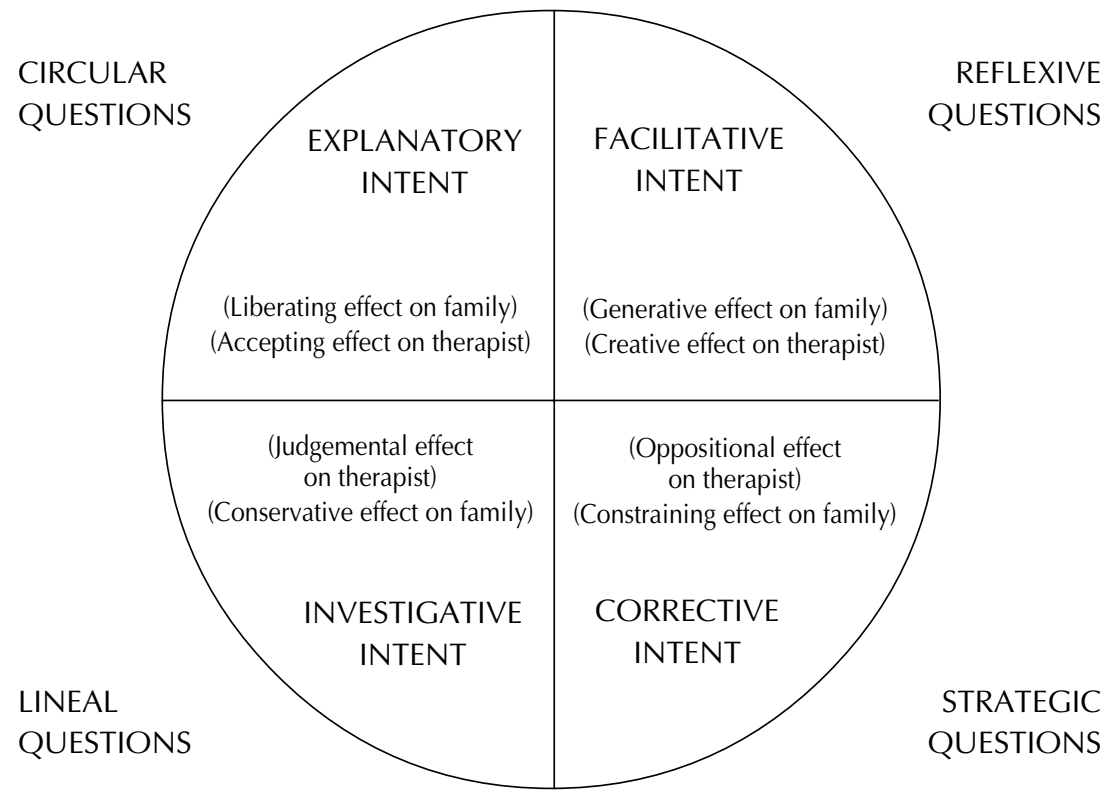

Tiré de Karl Tomm, Family Process, vol. 27, mars 1988, p. 13. 